\title{
Duas Aproximações para a DCT Baseadas na Fatoração de Chen
}

\author{
C. J. Tablada, F. M. Bayer e R. J. Cintra
}

\begin{abstract}
Resumo-Baseadas na fatoração de Chen, duas novas aproximações para a DCT são propostas. As aproximações propostas são livres de multiplicação e têm algoritmos rápidos eficientes. Tais aproximações foram avaliadas em termos de custo computacional e medidas de erro, sendo melhores aproximações para a DCT de comprimento 8 do que a signed DCT, a transformadas de Hadamard, a transformada de Walsh-Hadamard e a aproximação de Bouguezel-Ahmad-Swamy, em termos de erro de energia. Os métodos propostos chegam a requerer apenas 22 adições e são adequados como ferramentas de compressão de imagens.
\end{abstract}

Palavras-Chave-DCT aproximada; Compressão de imagens; Fatoração de Chen.

Abstract-Two multiplication-free DCT approximations based on Chen's factorization are proposed and their associate fast algorithms are also derived. The proposed transforms are assessed by means of computational cost and error measures; and are shown to better approximate the 8-point DCT, in terms of energy error, when compared with the signed DCT, the Hadamard transform, the Walsh-Hadamard transform, and the BouguezelAhmad-Swamy approximation. The proposed methods require as few as 22 additions and are adequate as image compression tools.

Keywords-Approximate DCT; Image compression; Chen's factorization.

\section{INTRODUÇÃO}

Transformadas discretas tem um papel central em técnicas de processamento de sinais e técnicas de compressão [7], [15]. Neste contexto, a transformada discreta do cosseno (DCT) é uma ferramenta crucial [2] pelo fato de ser uma prática aproximação para a transformada de Karhunen-Loève (KLT) [12]. A KLT tem a distinção de ser ótima em termos de compactação de energia quando os sinais processados são bem modelados por um processo markoviano de primeiro ordem com alta correlação [7]. Esta é suposição frequentemente satisfeita em imagens naturais [13].

Em particular, a DCT tipo II (DCT-II) de comprimento 8 tem ampla utilização em compressão de imagens [7], sendo adotada em diversos padrões de compressão, tais como JPEG [13], MPEG-1, MPEG-2, H.261, H.263 [8] e H.264 [20]. Objetivando o cálculo eficiente da DCT, muitos algoritmos rápidos vem sendo desenvolvidos [9], [19], [22], [28]. Entretanto, estes métodos usualmente requerem operações de elevado custo computacional, como a multiplicação, e uma

C. J. Tablada, Programa de Pós-graduação em Estatística, Universidade Federal de Pernambuco. F. M. Bayer, Departamento de Estatística, Laboratório de Ciências Espaciais de Santa Maria (LACESM), Universidade Federal de Santa Maria. R. J. Cintra, Departamento de Estatística, Programa de Pós-graduação em Engenharia Elétrica e Programa de Pós-graduação em Estatística, Universidade Federal de Pernambuco. E-mail: rjdsc@ieee.org Este trabalho foi parcialmente financiado pelo CNPq, CAPES e FACEPE. aritmética de ponto flutuante, que demanda maiores exigências de hardware [18].

Uma possível alternativa ao cálculo exato da DCT é o uso de aproximações que operem em aritmética inteira e não exijam multiplicações [7], [15]. Nesse contexto, diversas aproximações para a DCT-II foram propostas em que os elementos da matriz de transformação são definidos sobre o conjunto $\mathcal{P}=\left\{0, \pm \frac{1}{2}, \pm 1, \pm 2\right\}$ [3]-[6], [11], [14]. Métodos relevantes incluem a signed DCT (SDCT) [14] e a aproximação de Bouguezel-Ahmad-Swamy (BAS) [4]. Por construção, matrizes com elementos em $\mathcal{P}$ resultam em transformadas com complexidade multiplicativa nula, uma vez que as operações envolvidas podem ser implementadas por meio de adições e deslocamentos de bits (bit shifting) [3]. Estas aproximadas não senoidais podem ser usadas para implementações hardwaresoftware em diversos dispositivos computacionais de baixo poder de processamento [8], [20], [21].

No presente trabalho, temos as seguintes contribuições: (i) propomos duas novas aproximações para a DCT de comprimento 8, baseadas no algoritmo de Chen et al. [9] para a DCT exata; (ii) derivamos algoritmos rápidos para as transformadas propostas; (iii) avaliamos as aproximações propostas por meio de figuras de mérito; e (iv) comparamos as técnicas propostas com as aproximações SDCT e BAS, além das transformadas de Hadamard e Walsh-Hadamard [15], por serem livres de multiplicação.

\section{FATORAÇÃO DE CHEN PARA A DCT}

Em [9], Chen et al. propuseram uma fatoração para DCTII baseada numa fatoração para DCT de tipo IV (DCT-IV). Estas duas versões da DCT diferem entre si nos pontos de amostragem da função cosseno utilizados na definição de suas matrizes de transformação [7], [25]. Tais matrizes de transformação, respectivamente denotadas por $\mathbf{C}_{N}^{\mathrm{II}}$ e $\mathbf{C}_{N}^{\mathrm{IV}}$, são dadas por:

$$
\begin{aligned}
{\left[\mathbf{C}_{N}^{\mathrm{II}}\right]_{k, n} } & =\left[\sqrt{\frac{2}{N}} c_{k} \cos \left(\frac{k(2 n+1) \pi}{2 N}\right)\right]_{k, n}, \\
{\left[\mathbf{C}_{N}^{\mathrm{IV}}\right]_{k, n} } & =\left[\cos \left(\frac{(2 k+1)(2 n+1) \pi}{4 N}\right)\right]_{k, n},
\end{aligned}
$$

em que $k, n=0,1, \ldots, N-1, c_{0}=1 / \sqrt{2}, c_{k}=1$, se $k \neq 0$.

Sejam $\mathbf{I}_{N}$ a matriz identidade de ordem $N$ e $\overline{\mathbf{I}}_{N}$ a seguinte matriz de ordem $N$ :

$$
\overline{\mathbf{I}}_{N}=\left[\begin{array}{cccc}
0 & \cdots & 0 & 1 \\
0 & \cdots & 1 & 0 \\
\vdots & \ddots & \vdots & \vdots \\
1 & \cdots & 0 & 0
\end{array}\right] .
$$


Em [24], Wang demonstrou que a DCT-II de comprimento 8 admite a seguinte fatoração:

$$
\mathbf{C}_{8}^{\mathrm{II}}=\frac{1}{2} \mathbf{P}_{8} \cdot\left[\begin{array}{l|l}
\mathbf{C}_{4}^{\mathrm{II}} & \\
\hline & \overline{\mathbf{I}}_{4} \cdot \mathbf{C}_{4}^{\mathrm{IV}} \cdot \overline{\mathbf{I}}_{4}
\end{array}\right] \cdot \mathbf{B}_{8},
$$

em que $\mathbf{P}_{8}$ e $\mathbf{B}_{8}$ são matrizes de permutação e pré-adição, respectivamente, dadas por:

$$
\mathbf{P}_{8}=\left[\begin{array}{llllllll}
1 & 0 & 0 & 0 & 0 & 0 & 0 & 0 \\
0 & 0 & 0 & 0 & 0 & 0 & 0 & 1 \\
0 & 1 & 0 & 0 & 0 & 0 & 0 & 0 \\
0 & 0 & 0 & 0 & 0 & 0 & 1 & 0 \\
0 & 0 & 1 & 0 & 0 & 0 & 0 & 0 \\
0 & 0 & 0 & 0 & 0 & 1 & 0 & 0 \\
0 & 0 & 0 & 1 & 0 & 0 & 0 & 0 \\
0 & 0 & 0 & 0 & 1 & 0 & 0 & 0
\end{array}\right], \quad \mathbf{B}_{8}=\left[\begin{array}{c|c}
\mathbf{I}_{4} & \overline{\mathbf{I}}_{4} \\
\hline \overline{\mathbf{I}}_{4} & -\mathbf{I}_{4}
\end{array}\right] .
$$

Em [9], Chen et al. sugere que a matriz $\mathbf{C}_{4}^{\mathrm{IV}}$ admite a seguinte fatoração:

$$
\mathbf{C}_{4}^{\mathrm{IV}}=\mathbf{Q} \cdot \mathbf{A}_{1} \cdot \mathbf{A}_{2} \cdot \mathbf{A}_{3}
$$

em que

$$
\begin{aligned}
\mathbf{Q} & =\left[\begin{array}{llll}
1 & 0 & 0 & 0 \\
0 & 0 & 1 & 0 \\
0 & 1 & 0 & 0 \\
0 & 0 & 0 & 1
\end{array}\right], \mathbf{A}_{1}=\left[\begin{array}{rrrr}
\beta_{0} & 0 & 0 & \beta_{3} \\
0 & \beta_{2} & \beta_{1} & 0 \\
0 & \beta_{1} & -\beta_{2} & 0 \\
\beta_{3} & 0 & 0 & -\beta_{0}
\end{array}\right], \\
\mathbf{A}_{2} & =\left[\begin{array}{rrrr}
1 & 1 & 0 & 0 \\
1 & -1 & 0 & 0 \\
0 & 0 & -1 & 1 \\
0 & 0 & 1 & 1
\end{array}\right], \mathbf{A}_{3}=\left[\begin{array}{rrrr}
0 & 0 & 0 & 1 \\
0 & \alpha & \alpha & 0 \\
0 & -\alpha & \alpha & 0 \\
1 & 0 & 0 & 0
\end{array}\right],
\end{aligned}
$$

em que $\alpha=\cos \left(\frac{\pi}{4}\right)$ e $\beta_{n}=\cos \left(\frac{(2 n+1) \pi}{16}\right)$.

Substituindo (2) em (1) e expandindo a fatoração, obtemos

$$
\mathbf{C}_{8}^{\mathrm{II}}=\frac{1}{2} \cdot \mathbf{P}_{8} \cdot \mathbf{M}_{1} \cdot \mathbf{M}_{2} \cdot \mathbf{M}_{3} \cdot \mathbf{M}_{4} \cdot \mathbf{B}_{8}
$$

em que

$$
\begin{aligned}
\mathbf{M}_{1} & =\left[\begin{array}{l|l}
\mathbf{I}_{4} & \\
\hline & \overline{\mathbf{I}}_{4} \cdot \mathbf{Q}
\end{array}\right], \mathbf{M}_{2}=\left[\begin{array}{l|l|l}
\mathbf{P}_{4} & \\
\hline & & \mathbf{A}_{1}
\end{array}\right], \mathbf{M}_{3}=\left[\begin{array}{l|l}
\widetilde{\mathbf{C}} & \\
\hline & \mathbf{A}_{2}
\end{array}\right], \\
\mathbf{M}_{4} & =\left[\begin{array}{l|l}
\mathbf{B}_{4} & \\
\hline & \mathbf{A}_{3}
\end{array}\right], \mathbf{P}_{4}=\left[\begin{array}{llll}
1 & 0 & 0 & 0 \\
0 & 0 & 0 & 1 \\
0 & 1 & 0 & 0 \\
0 & 0 & 1 & 0
\end{array}\right], \mathbf{B}_{4}=\left[\begin{array}{l|l}
\mathbf{I}_{2} & \overline{\mathbf{I}}_{2} \\
\hline \overline{\mathbf{I}}_{2} & -\mathbf{I}_{2}
\end{array}\right], \\
\widetilde{\mathbf{C}} & =\left[\begin{array}{l|l}
\mathbf{C}_{2}^{\mathrm{II}} & \\
\hline & \overline{\mathbf{I}}_{2} \cdot \mathbf{C}_{2}^{\mathrm{IV}} \cdot \overline{\mathbf{I}}_{2}
\end{array}\right]=\left[\begin{array}{cccc}
\alpha & \alpha & 0 & 0 \\
\alpha & -\alpha & 0 & 0 \\
0 & 0 & -\gamma_{0} & \gamma_{1} \\
0 & 0 & \gamma_{1} & \gamma_{0}
\end{array}\right]
\end{aligned}
$$

e $\gamma_{n}=\cos \left(\frac{(2 n+1) \pi}{8}\right)$. A expressão (3) é chamada de fatoração de Chen.

As quantidades $\alpha, \beta_{n}$ e $\gamma_{n}$ são irracionais presentes apenas nas matrizes $\mathbf{M}_{2}, \mathbf{M}_{3}$ e $\mathbf{M}_{4}$. Tais quantidades exigem operações de multiplicação usualmente sobre aritmética de ponto flutuante.

\section{APROXIMAÇÕES PROPOSTAS}

Nesta seção, novas aproximações para a DCT-II são introduzidas. Para tanto, manipularemos as matrizes $\mathbf{M}_{2}, \mathbf{M}_{3}$ e $\mathbf{M}_{4}$ da fatoração de Chen, visando derivar novas matrizes cujos elementos estejam definidos sobre o conjunto $\mathcal{P}=$ $\left\{0, \pm \frac{1}{2}, \pm 1, \pm 2\right\}$.

Inicialmente, consideremos as funções sinal e de arredondamento denotadas por:

$$
\operatorname{sign}(x)=\left\{\begin{array}{ll}
1, & \text { se } x>0, \\
0, & \text { se } x=0, \\
-1, & \text { se } x<0,
\end{array} \quad \text { e } \quad \text { round }(\cdot),\right.
$$

em que, para $x \in \mathbb{R}$, $\operatorname{round}(x)$ denota o inteiro mais próximo a $x$. Estas funções coincidem com suas definições implementadas na linguagem $\mathrm{C}$ e em MATLAB. As funções $\operatorname{sign}(\cdot)$ e round $(\cdot)$, quando operadas sobre matrizes, operam elemento a elemento.

Desse modo, aplicando diretamente as funções acima, obtemos as seguintes novas matrizes:

$$
\widetilde{\mathbf{M}}_{i}=\operatorname{sign}\left(\mathbf{M}_{i}\right) \text { e } \widehat{\mathbf{M}}_{i}=\operatorname{round}\left(\mathbf{M}_{i}\right), \quad i=2,3,4 .
$$

Considerando as definições das matrizes $\mathbf{M}_{i}$, temos que:

$$
\begin{aligned}
& \widetilde{\mathbf{M}}_{2}=\left[\begin{array}{l|l}
\mathbf{P}_{4} & \\
\hline & \operatorname{sign}\left(\mathbf{A}_{1}\right)
\end{array}\right], \widetilde{\mathbf{M}}_{3}=\left[\begin{array}{l|l}
\operatorname{sign}(\tilde{\mathbf{C}}) & \\
\hline & \mathbf{A}_{2}
\end{array}\right], \\
& \widetilde{\mathbf{M}}_{4}=\left[\begin{array}{l|l}
\mathbf{B}_{4} & \\
\hline & \operatorname{sign}\left(\mathbf{A}_{3}\right)
\end{array}\right], \widehat{\mathbf{M}}_{2}=\left[\begin{array}{l|l}
\mathbf{P}_{4} & \\
\hline & \operatorname{round}\left(\mathbf{A}_{1}\right)
\end{array}\right], \\
& \widehat{\mathbf{M}}_{3}=\left[\begin{array}{l|l}
\operatorname{round}(\widetilde{\mathbf{C}}) & \\
\hline & \mathbf{A}_{2}
\end{array}\right], \widehat{\mathbf{M}}_{4}=\widetilde{\mathbf{M}}_{4} .
\end{aligned}
$$

Consequentemente, invocando a fatoração de Chen em (3), definimos as fatorações

$$
\begin{aligned}
& \widetilde{\mathbf{C}}_{8}=\frac{1}{2} \widetilde{\mathbf{T}}_{8} \cdot \widetilde{\mathbf{M}}_{3} \cdot \widetilde{\mathbf{M}}_{4} \cdot \mathbf{B}_{8}, \\
& \widehat{\mathbf{C}}_{8}=\frac{1}{2} \widehat{\mathbf{T}}_{8} \cdot \widehat{\mathbf{M}}_{3} \cdot \widehat{\mathbf{M}}_{4} \cdot \mathbf{B}_{8},
\end{aligned}
$$

em que $\widetilde{\mathbf{T}}_{8}=\mathbf{P}_{8} \cdot \mathbf{M}_{1} \cdot \widetilde{\mathbf{M}}_{2}$ e $\widehat{\mathbf{T}}_{8}=\mathbf{P}_{8} \cdot \mathbf{M}_{1} \cdot \widehat{\mathbf{M}}_{2}$. As matrizes $\widetilde{\mathbf{T}}_{8}$ e $\widehat{\mathbf{T}}_{8}$ são introduzidas explicitamente por brevidade de notação. As matrizes nas fatorações (4) e (5) têm inversas simples dadas por

$$
\begin{aligned}
& \widetilde{\mathbf{T}}_{8}^{-1}=\frac{1}{2}\left[\begin{array}{rrrrrrrr}
2 & 0 & 0 & 0 & 0 & 0 & 0 & 0 \\
0 & 0 & 0 & 0 & 2 & 0 & 0 & 0 \\
0 & 0 & 0 & 0 & 0 & 0 & 2 & 0 \\
0 & 0 & 2 & 0 & 0 & 0 & 0 & 0 \\
0 & 1 & 0 & 0 & 0 & 0 & 0 & 1 \\
0 & 0 & 0 & 1 & 0 & 1 & 0 & 0 \\
0 & 0 & 0 & -1 & 0 & 1 & 0 & 0 \\
0 & 1 & 0 & 0 & 0 & 0 & 0 & -1
\end{array}\right], \widetilde{\mathbf{M}}_{3}^{-1}=\frac{1}{2}\left[\begin{array}{rrrrrrrr}
1 & 1 & 0 & 0 & 0 & 0 & 0 & 0 \\
1 & -1 & 0 & 0 & 0 & 0 & 0 & 0 \\
0 & 0 & -1 & 1 & 0 & 0 & 0 & 0 \\
0 & 0 & 1 & 1 & 0 & 0 & 0 & 0 \\
0 & 0 & 0 & 0 & 1 & 1 & 0 & 0 \\
0 & 0 & 0 & 0 & 1 & -1 & 0 & 0 \\
0 & 0 & 0 & 0 & 0 & 0 & -1 & 1 \\
0 & 0 & 0 & 0 & 0 & 0 & 1 & 1
\end{array}\right], \\
& \widetilde{\mathbf{M}}_{4}^{-1}=\frac{1}{2}\left[\begin{array}{rrrrrrrr}
1 & 0 & 0 & 1 & 0 & 0 & 0 & 0 \\
0 & 1 & 1 & 0 & 0 & 0 & 0 & 0 \\
0 & 1 & -1 & 0 & 0 & 0 & 0 & 0 \\
1 & 0 & 0 & -1 & 0 & 0 & 0 & 0 \\
0 & 0 & 0 & 0 & 0 & 0 & 0 & 2 \\
0 & 0 & 0 & 0 & 0 & 1 & -1 & 0 \\
0 & 0 & 0 & 0 & 0 & 1 & 1 & 0 \\
0 & 0 & 0 & 0 & 2 & 0 & 0 & 0
\end{array}\right], \widehat{\mathbf{T}}_{8}^{-1}=\frac{1}{2}\left[\begin{array}{rrrrrrrr}
2 & 0 & 0 & 0 & 0 & 0 & 0 & 0 \\
0 & 0 & 0 & 0 & 2 & 0 & 0 & 0 \\
0 & 0 & 0 & 0 & 0 & 0 & 2 & 0 \\
0 & 0 & 2 & 0 & 0 & 0 & 0 & 0 \\
0 & 2 & 0 & 0 & 0 & 0 & 0 & 0 \\
0 & 0 & 0 & 1 & 0 & 1 & 0 & 0 \\
0 & 0 & 0 & -1 & 0 & 1 & 0 & 0 \\
0 & 0 & 0 & 0 & 0 & 0 & 0 & -2
\end{array}\right], \\
& \widehat{\mathbf{M}}_{3}^{-1}=\frac{1}{2}\left[\begin{array}{rrrrrrrr}
1 & 1 & 0 & 0 & 0 & 0 & 0 & 0 \\
1 & -1 & 0 & 0 & 0 & 0 & 0 & 0 \\
0 & 0 & -2 & 0 & 0 & 0 & 0 & 0 \\
0 & 0 & 0 & 2 & 0 & 0 & 0 & 0 \\
0 & 0 & 0 & 0 & 1 & 1 & 0 & 0 \\
0 & 0 & 0 & 0 & 1 & -1 & 0 & 0 \\
0 & 0 & 0 & 0 & 0 & 0 & -1 & 1 \\
0 & 0 & 0 & 0 & 0 & 0 & 1 & 1
\end{array}\right], \quad \widehat{\mathbf{M}}_{4}^{-1}=\widetilde{\mathbf{M}}_{4}^{-1}
\end{aligned}
$$

Note que a matriz $\mathbf{B}_{8}$ satisfaz $\mathbf{B}_{8} \cdot \mathbf{B}_{8}^{\mathrm{T}}=2 \mathbf{I}_{8}$. Assim, as aproximações para a DCT-II dadas por (4) e (5), têm inversas

$$
\begin{aligned}
& \widetilde{\mathbf{C}}_{8}^{-1}=\mathbf{B}_{8}^{\mathrm{T}} \cdot \widetilde{\mathbf{M}}_{4}^{-1} \cdot \widetilde{\mathbf{M}}_{3}^{-1} \cdot \widetilde{\mathbf{T}}_{8}^{-1}, \\
& \widehat{\mathbf{C}}_{8}^{-1}=\mathbf{B}_{8}^{\mathrm{T}} \cdot \widehat{\mathbf{M}}_{4}^{-1} \cdot \widehat{\mathbf{M}}_{3}^{-1} \cdot \widehat{\mathbf{T}}_{8}^{-1} .
\end{aligned}
$$

As aproximações propostas pertencem à classe de transformadas aproximadas não-ortogonais, tais como a SDCT [14] e a BAS [4]. Temos que, por construção, os elementos das matrizes envolvidas nas fatorações (4), (5), (6) e (7) pertencem ao conjunto $\mathcal{P}$. Dado que as transformadas $\widetilde{\mathbf{C}}_{8}$ e $\widehat{\mathbf{C}}_{8}$, bem como suas inversas, são implementadas através das respectivas fatorações, suas implementações são livres de multiplicações. 
Calculando as fatorações (4) e (5), obtemos explicitamente as seguintes aproximações para a DCT-II:

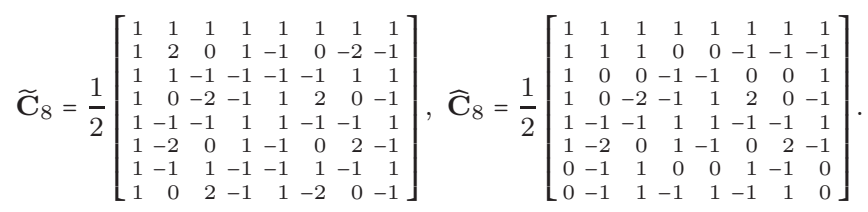

Por simplicidade de notação, no restante deste documento referenciamos a DCT-II simplesmente como a DCT.

\section{Medida de PROXimidade e Custo computacional}

Com o objetivo de mensurar a proximidade das novas aproximações propostas com relação a DCT exata, utilizamos o erro em energia como critério. Para $m, n=0,1, \ldots, 7$, sejam os sinais discretos $h_{m}[n]$ e $\hat{h}_{m}[n]$, formados pelas $(m+1, n+1)$-ésimas entradas das matrizes da DCT exata e de uma dada aproximação para a DCT, respectivamente. Tomando a transformada discreta de Fourier de tempo discreto destes sinais, obtemos $H_{m}(\omega)$ e $\widehat{H}_{m}(\omega), m=0,1, \ldots, 7$, que representam as funções de transferência das $(m+1)$ ésimas linhas das matrizes DCT exata e DCT aproximada, respectivamente. Assim, definimos o erro de energia da $(m+$ 1)-ésima linha matricial por [11]

$$
\epsilon_{m}=\int_{0}^{\pi}\left|H_{m}(\omega)-\widehat{H}_{m}(\omega)\right|^{2} \mathrm{~d} \omega
$$

O erro de energia total é simplesmente fornecido por $\epsilon_{\text {total }}=$ $\sum_{m=0}^{7} \epsilon_{m}$. Como descrito em [11], $\epsilon_{\text {total }}$ é uma medida de similaridade entre a DCT exata e a DCT aproximada considerada. Alternativamente, notamos que (8) pode ser calculada de $h_{m}[n]$ e $\hat{h}_{m}[n]$ pelo Teorema de Parseval [17, p. 18].

Seguindo a técnica proposta em [10], um fator de escalonamento de $\frac{1}{\sqrt{2}}$ é capaz de melhorar a aproximação das transformadas propostas $\widetilde{\mathbf{C}}_{8}$ e $\widehat{\mathbf{C}}_{8}$. Isto permite obter um menor erro de energia total. Em particular, obtemos que $\epsilon_{0}=$ $\epsilon_{4}=0$ (cf. Tabela I).

Portanto, (4) e (5) e suas inversas (6) e (7) resultam em:

$$
\begin{aligned}
\widetilde{\mathbf{C}}_{8} & =\frac{1}{2 \sqrt{2}} \widetilde{\mathbf{T}}_{8} \cdot \widetilde{\mathbf{M}}_{3} \cdot \widetilde{\mathbf{M}}_{4} \cdot \mathbf{B}_{8}, \\
\widehat{\mathbf{C}}_{8} & =\frac{1}{2 \sqrt{2}} \widehat{\mathbf{T}}_{8} \cdot \widehat{\mathbf{M}}_{3} \cdot \widehat{\mathbf{M}}_{4} \cdot \mathbf{B}_{8}, \\
\widetilde{\mathbf{C}}_{8}^{-1} & =\sqrt{2} \mathbf{B}_{8}^{\mathrm{T}} \cdot \widetilde{\mathbf{M}}_{4}^{-1} \cdot \widetilde{\mathbf{M}}_{3}^{-1} \cdot \widetilde{\mathbf{T}}_{8}^{-1}, \\
\widehat{\mathbf{C}}_{8}^{-1} & =\sqrt{2} \mathbf{B}_{8}^{\mathrm{T}} \cdot \widehat{\mathbf{M}}_{4}^{-1} \cdot \widehat{\mathbf{M}}_{3}^{-1} \cdot \widehat{\mathbf{T}}_{8}^{-1} .
\end{aligned}
$$

No contexto de compressão de imagens, estes fatores de escalonamento podem ser absorvidos na etapa de quantização [3], [11]. Assim, nesse sentido, não representam aumento na complexidade computacional.

Para comparação com as transformadas propostas, consideramos as clássicas aproximações SDCT e BAS—que são nãoortogonais, assim como as transformadas de Hadamard (HT) e Walsh-Hadamard (WHT), ambas ortogonais. Os resultados para a medida de erro de energia para as aproximações discutidas estão listadas na Tabela I. Adicionalmente, na Tabela II, são fornecidas as complexidades computacionais de cada método considerado neste trabalho.
TABELA I

ERRO DE ENERGIA

\begin{tabular}{ccccccc}
\hline$m$ & $\widehat{\mathbf{C}}_{8}$ & $\widetilde{\mathbf{C}}_{8}$ & SDCT & BAS & WHT & HT \\
\hline 0 & 0 & 0 & 0 & 0 & 0 & 0 \\
1 & 0.24 & 1.55 & 0.59 & 0.24 & 0.59 & 5.15 \\
2 & 0.61 & 0.48 & 0.48 & 0.48 & 0.48 & 6.28 \\
3 & 0.42 & 0.42 & 0.59 & 0.69 & 1.46 & 6.28 \\
4 & 0 & 0 & 0 & 0 & 0 & 6.28 \\
5 & 0.42 & 0.42 & 0.59 & 1.65 & 1.46 & 6.28 \\
6 & 0.61 & 0.48 & 0.48 & 0.48 & 0.48 & 8.69 \\
7 & 0.24 & 1.55 & 0.59 & 0.59 & 0.59 & 8.64 \\
\hline Total & 2.52 & 4.88 & 3.32 & 4.12 & 5.05 & 47.61 \\
\hline
\end{tabular}

TABELA II

CUSTO COMPUTACIONAL

\begin{tabular}{lcccc}
\hline & Adições & Mult. & Deslocamentos & Total \\
\cline { 2 - 5 } DCT exata & 26 & 16 & 0 & 42 \\
$\widehat{\mathbf{C}}_{8}$ & 22 & 0 & 0 & 22 \\
$\widetilde{\mathbf{C}}_{8}$ & 26 & 0 & 0 & 26 \\
SDCT & 24 & 0 & 0 & 24 \\
BAS & 21 & 0 & 0 & 21 \\
WHT & 24 & 0 & 0 & 24 \\
HT & 24 & 0 & 0 & 24 \\
\hline
\end{tabular}

A aproximação proposta $\widehat{\mathbf{C}}_{8}$ apresenta o menor erro de energia entre as transformadas consideradas e requer apenas 22 adições, i.e., duas operações a menos que a SDCT e apenas uma a mais que a BAS. A Tabela II também apresenta o custo computacional da DCT exata utilizando a fatoração de Chen descrita em (3), necessitando 26 adições e 16 multiplicações para sua implementação.

$\mathrm{O}$ custo computacional da aproximação $\widetilde{\mathbf{C}}_{8}$ é superior ao custo computacional das outras aproximações analisadas. A Figura 1 apresenta o diagrama de fluxo do algoritmo rápido proposto para a aproximação $\widehat{\mathbf{C}}_{8}$. $\mathrm{O}$ digrama para a aproximação $\widetilde{\mathbf{C}}_{8}$ é similar e foi suprimido por brevidade.

\section{APliCAÇÕES EM COMPRESSÃO DE IMAGENS}

\section{A. Compressão tipo JPEG e medidas de erro}

As aproximações $\widetilde{\mathbf{C}}_{8}$ e $\widehat{\mathbf{C}}_{8}$ propostas foram avaliadas no contexto de compressão de imagens, conjuntamente com as aproximações SDCT, BAS, HT e WHT. Para tanto, foi usado

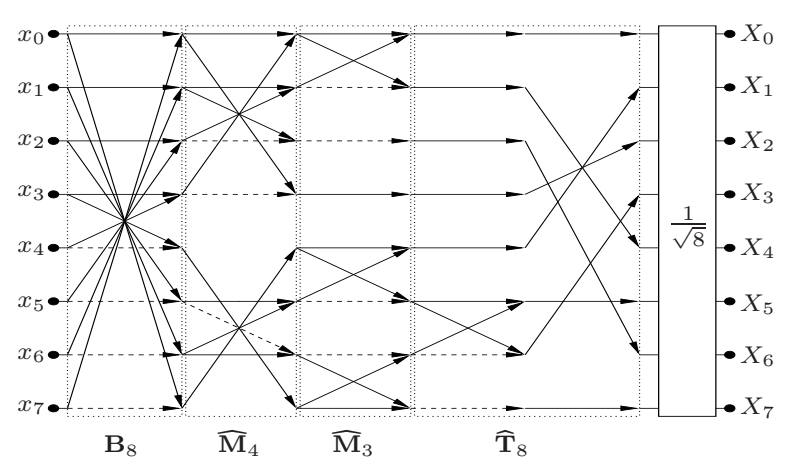

Fig. 1. Diagrama de fluxo para a aproximação $\widehat{\mathbf{C}}_{8}$ (as linhas em traços indicam multiplicação por -1 ). 
um conjunto de 30 imagens padronizadas de 8 -bits em escala de cinza de tamanho $512 \times 512$ obtidas de [1]. Cada imagem foi subdividida em blocos de tamanho $8 \times 8$. Cada bloco $\mathbf{B}$ foi submetido à transformada 2-D, da seguinte forma [23]

$$
\mathbf{T} \cdot \mathbf{B} \cdot \mathbf{T}^{-1}
$$

em que $\mathbf{T}$ é a matriz de uma das transformadas consideradas. Os 64 coeficientes resultantes para cada bloco B foram ordenados segundo a sequência zig-zag padrão e apenas $r$ coeficientes foram retidos pelo processo de compressão. Como sugerido em [11], adotamos $1 \leq r \leq 45$. Finalmente, a transformada 2-D inversa é aplicada para reconstruir a imagem compactada. A imagem resultante é então comparada com a imagem original segundo medidas objetivas de qualidade.

Como medidas de avaliação foram consideradas a relação sinal-ruído de pico (PSNR) [16], o erro médio quadrático (MSE) [26] e o índice de similaridade estrutural (SSIM) [26], [27]. Para o MSE e o SSIM, também foram calculados os erros absolutos percentuais (EAP) relativos à DCT exata. Seguindo a metodologia de [11], a média das medidas de avaliação sob as 30 imagens padronizadas foram calculadas, o que resulta em resultados estatisticamente mais robustos, quando comparados com análises feitas em imagens particulares. As medidas de PSNR e MSE são comumente as mais utilizadas para comparar técnicas de compressão. Contudo, como apontado em [27], o SSIM considera características do sistema visual humano não consideradas nas medidas anteriores.

\section{B. Resultados}

Os valores resultantes dos experimentos computacionais são apresentados nas Figura 2(a)-(c). Na Figura 2(c), a curva correspondente a HT foi suprimida, pois apresenta valores excessivamente altos em comparação às outras. Os resultados mostram que, em termos de PSNR ou MSE, a aproximação $\widehat{\mathbf{C}}_{8}$ supera a SDCT e a BAS; e proporciona resultados similares à WHT, mas com um menor custo computacional. Em termos de SSIM, temos que as duas aproximações propostas $\widetilde{\mathbf{C}}_{8}$ e $\widehat{\mathbf{C}}_{8}$ tem desempenhos similares.

A aproximação proposta tem desempenho bastante próximo ao da WHT. Conforme as Figuras 2(a) e 2(b), o desempenho da transformada proposta $\widehat{\mathbf{C}}_{8}$ é superior ao desempenho da WHT para $r$ pequeno $(r \leq 15)$. Assim, na Figura 3, é apresentada a imagem padrão Elaine após o processo de compressão considerando apenas a WHT e a aproximação proposta $\widehat{\mathbf{C}}_{8}$. Imagens para a DCT exata e SDCT também são exibidas, por serem métodos de referência. Todas as imagens foram comprimidas utilizando $r=6$, valor que representa um truncamento de aproximadamente $90.5 \%$ dos coeficientes. A análise visual dessas imagens obtidas mostra a superioridade da transformada $\widehat{\mathbf{C}}_{8}$ proposta sobre a SDCT em compressão de imagens. Ainda, a Tabela III evidencia quantitativamente o melhor desempenho de $\widehat{\mathbf{C}}_{8}$ sobre as demais transformadas consideradas.

\section{Análise de blocking artifact}

Um efeito visual indesejável em compressão de imagens é o surgimento de blocos na imagem. Esse efeito é conhecido como blocking artifact [13, p. 573].

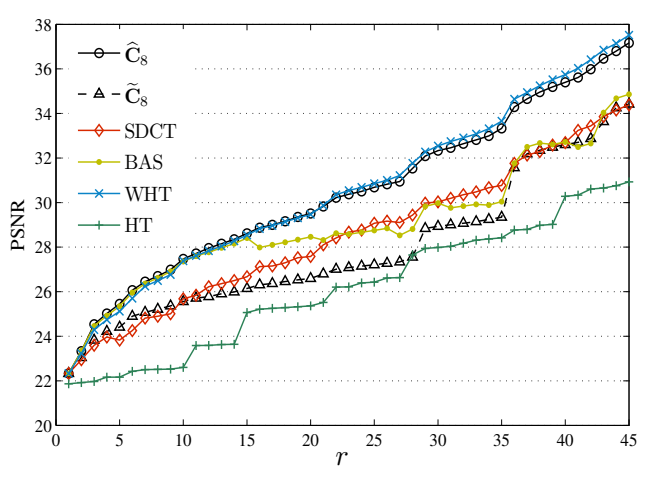

(a)

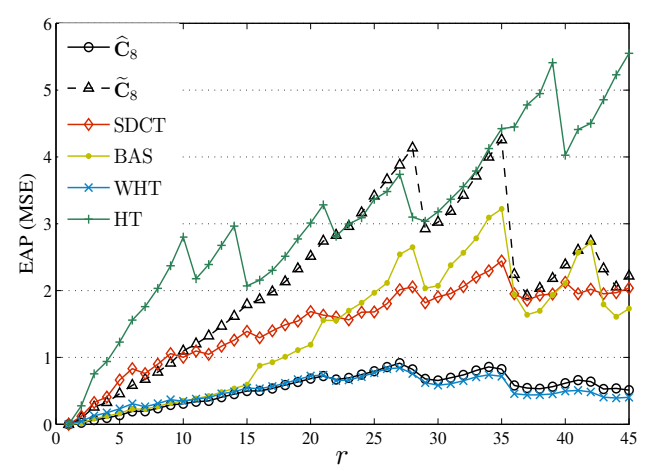

(b)

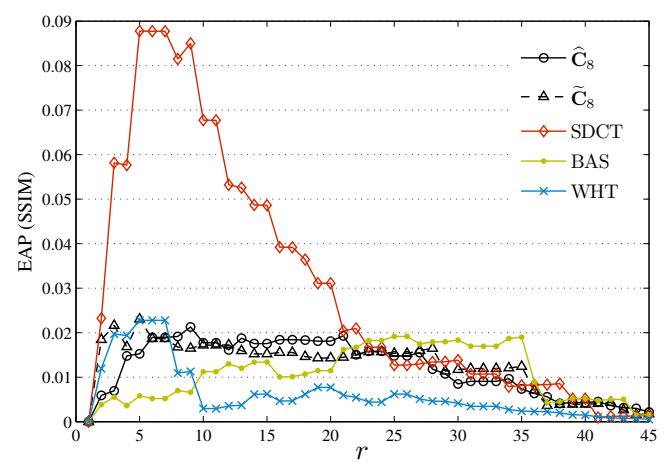

(c)

Fig. 2. Curvas para a média correspondente a 30 imagens. (a) PSNR, (b) EAP (MSE) e (c) EAP (SSIM).

TABELA III

MEDidAS DE AVALIAÇÃO DE COMPRESS ÃO DA IMAGEM ELA I NE, CONSIDERANDO $r=6$

\begin{tabular}{lccc}
\hline Transformada & PSNR & MSE & SSIM \\
\hline DCT exata & 31.03 & 51.33 & 0.95 \\
$\widehat{\mathbf{C}}_{8}$ & 30.00 & 65.00 & 0.94 \\
WHT & 28.91 & 83.53 & 0.92 \\
SDCT & 27.59 & 113.37 & 0.88 \\
\hline
\end{tabular}




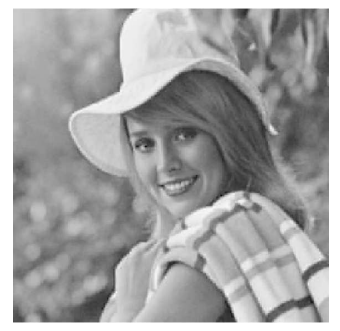

(a) DCT

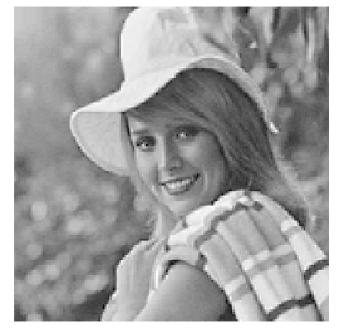

(c) SDCT

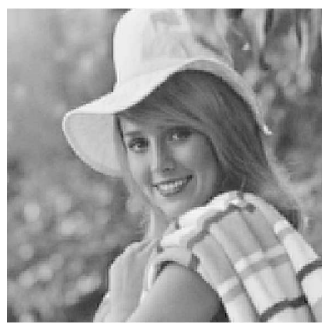

(b) WHT

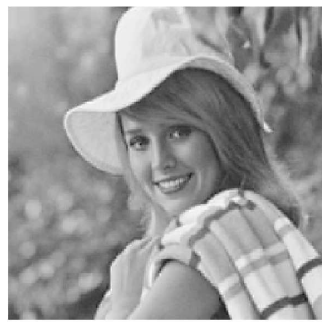

(d) $\widehat{\mathbf{C}}_{8}$
Fig. 3. Imagem Elaine $(r=6)$ : (a) DCT exata, (b) WHT, (c) SDCT e (d) $\widehat{\mathbf{C}}_{8}$.

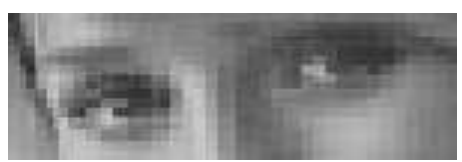

(a) WHT

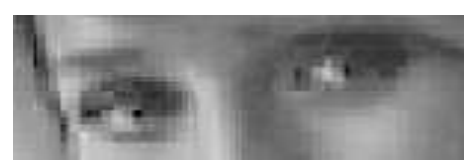

(b) $\widehat{\mathbf{C}}_{8}$

Fig. 4. Efeito de blocking artifact na imagem Elaine $(r=6)$ : (a) WHT e (b) $\widehat{\mathbf{C}}_{8}$.

A Figura 4 mostra qualitativamente essa comparação em termos de blocking artifact derivados da $\widehat{\mathbf{C}}_{8}$ e WHT. A utilização de $\widehat{\mathbf{C}}_{8}$ na compressão de imagens minimiza o aparecimento de blocking artifact comparativamente à WHT.

\section{CONCLUSÕES}

O presente trabalho apresenta duas aproximações para a DCT baseadas na fatoração de Chen. Seus desempenhos foram comparados com outras aproximações bastante conhecidas na literatura de processamento de sinais. A aproximação $\widehat{\mathbf{C}}_{8}$ apresentou o menor erro de energia total dentre todas as aproximações analisadas, evidenciando uma maior similaridade espectral com a DCT exata. A aproximação $\widehat{\mathbf{C}}_{8}$ também demostrou superioridade sobre a SDCT, a BAS e a HT em termos de PSNR e MSE das imagens comprimidas, tendo um desempenho similar à WHT, porém com um custo computacional $8 \%$ inferior.

\section{REFERÊNCIAS}

[1] The USC-SIPI Image Database, University of Southern California, Signal and Image Processing Institute., 2011.
[2] N. Ahmed, T. Natarajan, and K. R. Rao. Discrete Cosine Transform. IEEE Trans. Comput., C-23(1):90-93, January 1974.

[3] F. M. Bayer and R. J. Cintra. DCT-like transform for image compression requires 14 additions only. Electron. Lett., 48(15):919-921, July 2012.

[4] S. Bouguezel, M. O. Ahmad, and M. N. S. Swamy. A multiplicationfree transform for image compression. In 2nd International Conference on Signals, Circuits and Syst. (SCS), pages 1-4, November 2008.

[5] S. Bouguezel, M. O. Ahmad, and M. N. S. Swamy. A novel transform for image compression. In IEEE 53rd International Midwest Symposium on Circuits Syst. (MWSCAS), pages 509-512, August 2010.

[6] S. Bouguezel, M. O. Ahmad, and M. N. S. Swamy. A low-complexity parametric transform for image compression. In IEEE International Symposium on Circuits Syst. (ISCAS), pages 2145-2148, May 2011.

[7] V. Britanak, P. C. Yip, and K. R. Rao. Discrete Cosine and Sine Transforms: General Properties, Fast Algorithms and Integer Approximations. Elsevier Science, 2010.

[8] T. S. Chang, C. S. Kung, and C. W. Jen. A simple processor core design for DCT/IDCT. IEEE Trans. Circuits Syst. Video Technol., 10(3):439447, April 2000.

[9] W. H. Chen, C. Smith, and S. Fralick. A fast computational algorithm for the Discrete Cosine Transform. IEEE Trans. Commun., 25(9):10041009, September 1977.

[10] R. J. Cintra. An integer approximation method for discrete sinusoidal transforms. Circuits, Syst., and Signal Process., 30(6):1481-1501, December 2011.

[11] R. J. Cintra and F. M. Bayer. A DCT approximation for image compression. IEEE Signal Process. Lett., 18(10):579-582, October 2011.

[12] M. Effros, H. Feng, and K. Zeger. Suboptimality of the Karhunen-Loève transform for transform coding. IEEE Trans. Inf. Theory, 50(8):16051619, August 2004.

[13] R. C. Gonzalez and R. E. Woods. Digital image processing. PrenticeHall, Inc., Upper Saddle River, NJ, USA, 3rd edition, 2006.

[14] T. I. Haweel. A new square wave transform based on the DCT. Signal Process., 81(11):2309-2319, November 2001.

[15] K. J. Horadam. Hadamard matrices and their applications. Cryptography Commun., 2(2):129-154, 2010.

[16] Q. Huynh-Thu and M. Ghanbari. Scope of validity of PSNR in image/video quality assessment. Electron. Lett., 44(13):800-801, June 2008.

[17] Anil K. Jain. Fundamentals of digital image processing. Prentice-Hall, Inc., Upper Saddle River, N. J., USA, 1989.

[18] Jie Liang and T. D. Tran. Fast multiplierless approximations of the DCT with the lifting scheme. IEEE Trans. Signal Process., 49(12):3032-3044, December 2001.

[19] C. Loeffler, A. Ligtenberg, and G. S. Moschytz. Practical fast 1-D DCT algorithms with 11 multiplications. In International Conference on Acoust., Speech, Signal Process. (ICASSP), pages 988-991, May 1989.

[20] A. Puri, X. Chen, and A. Luthra. Video coding using the H.264/MPEG4 AVC compression standard. Signal Process.: Image Commun., 19(9):793-849, October 2004

[21] N. Roma and L. Sousa. Efficient hybrid DCT-domain algorithm for video spatial downscaling. EURASIP J. Adv. Signal Process, 2007(2):3030, June 2007.

[22] N. Suehiro and M. Hatori. Fast algorithms for the DFT and other sinusoidal transforms. IEEE Trans. Acoust., Speech, Signal Process., 34(3):642-644, June 1986.

[23] T. Suzuki and M. Ikehara. Integer DCT based on direct-lifting of DCTIDCT for lossless-to-lossy image coding. IEEE Trans. Image Process., 19(11):2958-2965, November 2010.

[24] Z. Wang. Reconsideration of: A fast computational algorithm for the Discrete Cosine Transform. IEEE Trans. Commun., 31(1):121-123, January 1983.

[25] Z. Wang. Fast algorithms for the discrete W transform and for the Discrete Fourier Transform. IEEE Trans. Acoust., Speech, Signal Process., 32(4):803-816, August 1984.

[26] Z. Wang and A. C. Bovik. Mean squared error: Love it or leave it? A new look at signal fidelity measures. IEEE Signal Process. Mag., 26(1):98-117, January 2009.

[27] Z. Wang, A. C. Bovik, H. R. Sheikh, and E. P. Simoncelli. Image quality assessment: from error visibility to structural similarity. IEEE Trans. Image Process., 13(4):600-612, April 2004.

[28] W. Yuan, P. Hao, and C. Xu. Matrix factorization for fast DCT algorithms. In IEEE International Conference on Acoust., Speech, Signal Process. (ICASSP), volume 3, pages 948-951, May 2006. 